\title{
Análise da transferência de calor em paredes compostas por diferentes materiais
}

\author{
Heat transfer analysis in walls composed by different \\ materials
}

\section{Luciano Pivoto Specht \\ Pedro Augusto Pereira Borges \\ Ricardo Forgiarini Rupp \\ Rosane Varnier}

\section{Resumo}

Luciano Pivoto Specht Pós-Graduação em Modelagem Matemática Universidade Regional do Noroeste do Estado do Rio Grande do Sul Campus ljuí Rua do Comércio, 3000 Ijuí - RS - Brasil CEP $98700-000$ Tel.: (55) 3332-0512

E-mail: luspecht@gmail.com

Pedro Augusto Pereira Borges Pós-Graduação em Modelagem Matemática Universidade Regional do Noroeste do Estado do Rio Grande do Sul E-mail: papborges@gmail.com

Ricardo Forgiarini Rupp Pós-Graduação em Modelagem Matemática

Universidade Regional do Noroeste do Estado do Rio Grande do Sul E-mail: ricardorupp@gmail.com

Rosane Varnier Pós-Graduação em Modelagem Matemática Universidade Regional do Noroeste do Estado do Rio Grande do Sul E-mail: rosane.varnierzanon@yahoo.com.br

Recebido em 15/02/10 Aceito em 21/07/10

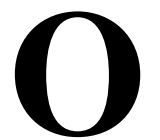

projeto de edificações energeticamente eficientes necessita de conhecimentos sobre a transferência de calor proveniente do ambiente externo, a fim de criar soluções que associem diferentes materiais e espessuras às condições desejadas de conforto térmico. O objetivo deste trabalho é avaliar diversos tipos e disposições de materiais em paredes de tijolos maciços, sob a ótica da térmica de edifícios. Para a realização dos experimentos, modelos em escala real foram confeccionados, instrumentados e acoplados a uma câmara térmica, desenvolvida especialmente para esse fim. O problema de transferência de calor foi modelado pela equação da energia, resolvida em Diferenças Finitas Centrais, utilizando-se o Método Implícito. As difusividades térmicas dos materiais foram calculadas resolvendo o Problema Inverso de transferência de calor. Também foi calculado o fluxo de calor e o custo total de cada alternativa, finalizando-se com a obtenção da relação termoeconômica para cada parede. Através da análise termoeconômica pode-se constatar que, apesar de o investimento inicial ser relativamente maior nas paredes mais espessas e/ou com isolamento térmico, estas apresentam um comportamento térmico muito superior às tradicionais. Notadamente, a parede com EPS na face exterior apresentou o melhor desempenho.

Palavras-chave: Transferência de calor. Paredes. Modelagem matemática. Eficiência energética.

\section{Abstract}

The project of efficient energy buildings requires knowledge about heat transfer from the external environment, in order to create solutions that combine different materials and thicknesses to the desired conditions of thermal comfort. The objective of this work is to assess, from de viewpoint of buildings physics, several types and materials arrangement on solid brick walls. For the achievement of the experiments, full-scale models were fabricated, instrumented and coupled to a heat chamber, developed especially for this purpose. The heat transfer problem was modelled by energy equation, solved in Central Finite Differences using the Implicit Method. The thermal diffusivities of the materials were calculated by solving the Inverse Problem of heat transfer. It was also calculated the heat flow and the total cost of each alternative, ending with the achievement of thermoeconomic relation to each wall. Through the thermo-economic analysis, it can be verified that although the initial investment is relatively higher on the thicker walls and/or with thermal insulation, these present a much higher thermal behaviour in comparison to the traditional ones. Notably, the wall with EPS on the external surface presented the best performance.

Keywords: Heat transfer. Walls. Mathematical modelling. Energy efficiency. 


\section{Introdução}

Diversos fatores têm contribuído para o aumento do uso de energia por todo o mundo. A globalização disseminou mundialmente o estilo de vida dos países mais desenvolvidos, levando muitas sociedades a um ponto extremo, onde não é possível reverter o quadro e viver sustentavelmente. Um desses fatores que causam grande impacto ambiental em nações desenvolvidas é o uso de energia em edificações. Globalmente, a indústria da construção e o ambiente construído são dois dos maiores consumidores de energia e materiais (SANTIN; ITARD; VISSCHER, 2009).

Na parte norte da União Europeia, conforme Itard e Meijer (2008), 41\% do total do consumo final de energia são gastos em edificações, sendo $30 \%$ utilizados em construções residenciais. De acordo com a EuroAce (2004), 57\% do consumo de energia em edificações são gastos com aquecimento de ambientes, $25 \%$ com aquecimento de água, $11 \%$ com iluminação e utensílios elétricos, e $7 \%$ com preparos de alimentos.

Já nos Estados Unidos o Buildings Energy Data Book (2006), publicado pelo Departamento de Energia, mostra que em 2006 o setor da construção consumiu $38,8 \%$ do total da energia primária utilizada no país. Dessa energia, $34,8 \%$ foram usados para aquecimento de ambientes, ventilação e ar condicionado (KWOK; RAJKOVICH, 2009).

No caso específico do Brasil, de acordo com o Procel (2009), cerca de $16 \%$ da produção nacional de energia elétrica são utilizados em edifícios comerciais, e $25 \%$ no setor residencial. De acordo com Lamberts, Dutra e Pereira (1997), estudos que mostram que $48 \%$ do total do consumo de energia elétrica em edificações comerciais são gastos com condicionamento de ar, podendo esse consumo chegar a $70 \%$ do total consumido em edifícios comerciais com fachadas envidraçadas. Já nas residências brasileiras o consumo de energia por meio da utilização de aparelhos de ar condicionado vem aumentando nos últimos anos, possivelmente devido ao aumento da renda do brasileiro e às condições inadequadas de desempenho térmico de suas moradias (PEÑA; GHISI; PEREIRA, 2008).

Um dos maiores problemas desse acréscimo do consumo mundial de energia é o aquecimento global. Na tentativa de reverter a atual situação, o Protocolo de Kyoto determina que os países signatários (na maioria países desenvolvidos) se comprometam a reduzir as emissões de gases do efeito estufa em pelo menos $5 \%$ (em relação aos níveis de 1990) no período entre 2008 e 2012.
Entre os métodos que os governos dispõem para atingir tal meta estão: melhorar a eficiência energética das edificações e os sistemas de energia; desenvolver conceitos de construções sustentáveis; e promover o uso de fontes renováveis de energia (CHLELA et al., 2007).

$\mathrm{O}$ projeto de edificações energeticamente eficientes necessita de conhecimentos sobre a transferência de calor do ambiente externo para o interior das edificações, a fim de criar soluções que associem diferentes materiais e dimensões de camadas às condições desejadas de conforto térmico. A produção desse conhecimento via construção de protótipos, além de onerosa, apresenta dificuldades com relação à variação dos materiais e dimensões das camadas. A pesquisa com modelagem matemática é uma alternativa que tem se mostrado eficiente, ágil e barata. Nessa alternativa a teoria de transferência de calor é associada a experimentos de laboratório, resultando em programas computacionais que podem ser usados como ferramenta de análise e projeto.

É prática comum na engenharia adotar soluções construtivas de menor custo econômico possível e de baixo investimento inicial. $\mathrm{Na}$ maioria das vezes, essas soluções geram construções de baixa qualidade energética, prejudicando o meio ambiente, além do que, no longo prazo, tornam-se mais onerosas do que soluções que levam em consideração outras variáveis, além da econômica.

O problema de transferência de calor em paredes compostas tem sido classicamente modelado utilizando-se a equação da energia, com condições de contorno lineares e em função do tempo (BECKETT; CHU, 1973; CHEN; LIN, 1991; SUN; JIA, 2007; HUANG; CHANG, 1980). A solução desse problema de condições de contorno tem sido implementada por diferentes métodos: Método da Transformada de Laplace (CARSLAW; JAEGER, 1959; CHONG; TRAMONTINI; SPECHT, 2009); Funções de Green (HUANG; CHANG, 1980); Método dos Elementos Finitos (SUGIYAMA; NISHIMURA; WATANBE, 1974; BECKETT; CHU, 1973); Método Híbrido: Transformada de Laplace e Elementos Finitos (CHEN; LIN, 1991). Todos esses métodos apresentam vantagens e desvantagens. Os métodos analíticos proporcionam soluções exatas e rápidas para qualquer tempo e espaço, mas a obtenção das inversas das transformadas (no caso da Transformada de Laplace) e a identificação de funções apropriadas (no caso da Função de Green) tornam a aplicação de tais métodos limitada. Os métodos numéricos, tais como o Método dos 
Elementos Finitos, Volumes Finitos e Diferenças Finitas (CHEN; LIN, 1991), têm sido amplamente utilizados com sucesso em problemas unidimensionais, com condições de contorno lineares e dependentes do tempo.

Dentro desse contexto, o presente trabalho busca soluções para melhorar a eficiência energética das edificações, tendo como objetivo: avaliar configurações diversas de paredes compostas por diferentes tipos e disposições de materiais sob a ótica da térmica em edifícios mediante ensaios de laboratório e modelagem matemática.

\section{Procedimentos experimentais e modelagem matemática}

A pesquisa foi executada em duas etapas: na primeira construiu-se um aparato experimental (câmara térmica, paredes e sistema de coleta de dados) para obter a variação da temperatura nas paredes; e na segunda o problema de transferência de calor foi modelado matematicamente, calculadas as difusividades térmicas e os fluxos de calor.

\section{Câmara térmica}

Com a finalidade de reproduzir aproximadamente as condições de aquecimento em paredes expostas ao sol, construiu-se uma câmara térmica (Figura 1), com dimensões internas de $60 \mathrm{~cm} \mathrm{x} 40 \mathrm{~cm} \mathrm{x} 40$ $\mathrm{cm}$. O equipamento é composto de placas de madeira, revestido com poliestireno expandido e papel laminado; no interior da câmara foi acoplada uma lâmpada incandescente, com potência $250 \mathrm{~W}$, modelo SICCATHERM (fonte de calor), cuja intensidade é controlada por um reostato. Ajustando manualmente a corrente elétrica, produziram-se as variações de temperatura na superfície da parede (superfície externa à edificação). Para medir a temperatura ao longo de cada parede utilizou-se um conjunto de termopares.

\section{Procedimentos experimentais}

Foram construídas sete paredes em alvenaria de tijolos maciços com diferentes configurações, conforme a Figura 2. Com exceção da Parede 1 (tijolo à vista), todas as estruturas possuem revestimento argamassado em ambas as faces, diferindo na espessura e/ou na disposição de material isolante térmico (poliestireno expandido). Essas estruturas foram construídas em laboratório, cada qual medindo cerca de $60 \mathrm{~cm}$ x $60 \mathrm{~cm}$. Em cada configuração de parede foram inseridos cinco sensores de temperatura ao longo da espessura L (eixo $\mathrm{x}$ ), para medidas no interior da parede; também foi instalado um sensor para medir a temperatura junto à superfície aquecida (lado externo da edificação, condição de fronteira em x $=0)$ e outro na superfície não aquecida (lado interno da edificação, condição de fronteira em x = L), totalizando, assim, sete sensores (RUPP, 2009).

\section{Modelagem matemática}

A modelagem matemática do problema em estudo foi realizada considerando-se as seguintes etapas: Caracterização do Problema Direto (equação da energia e condições de contorno); Solução do Problema Direto usando o Método das Diferenças Finitas; Problema Inverso (estimativa de propriedades térmicas); cálculo dos fluxos de calor nas superfícies interna e externa das paredes; e relação termoeconômica.

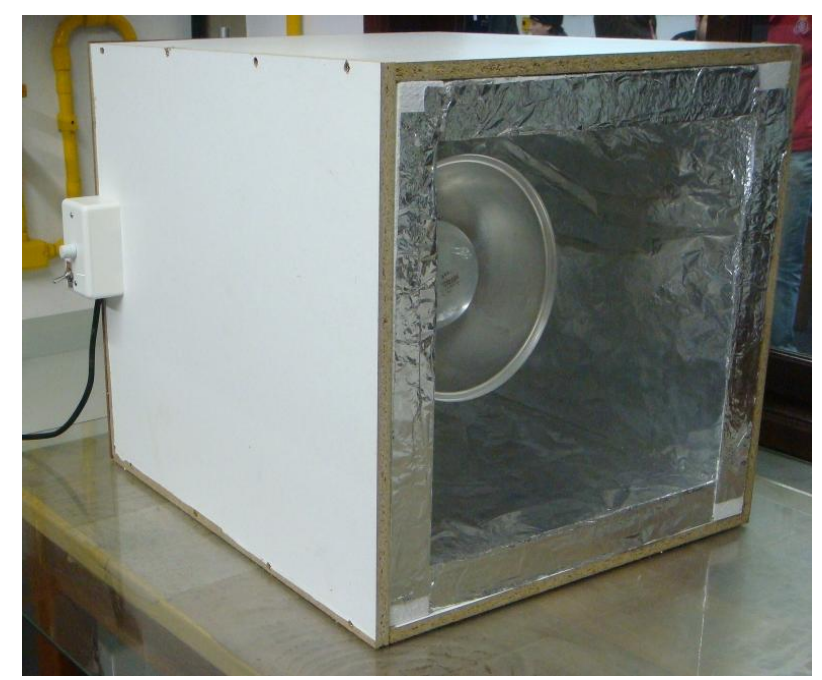

Figura 1 - Câmara térmica 


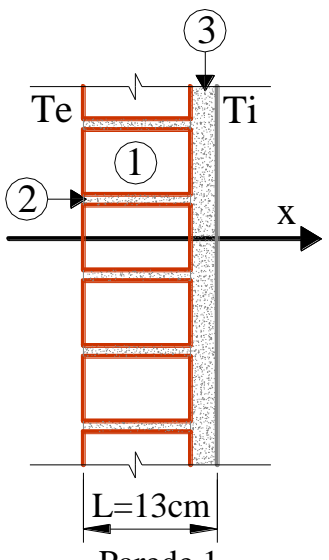

Parede 1
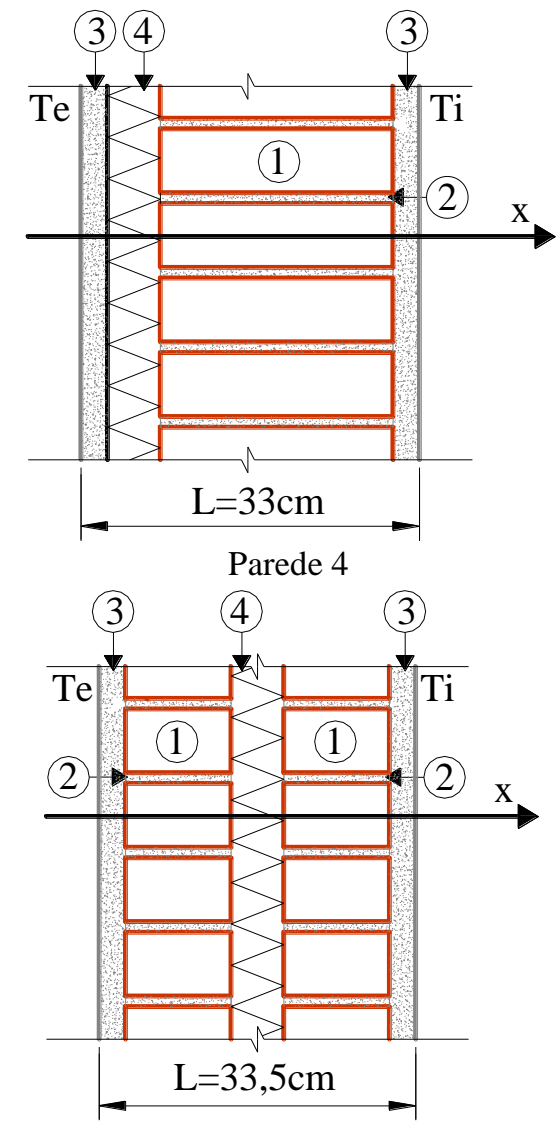

Parede 6

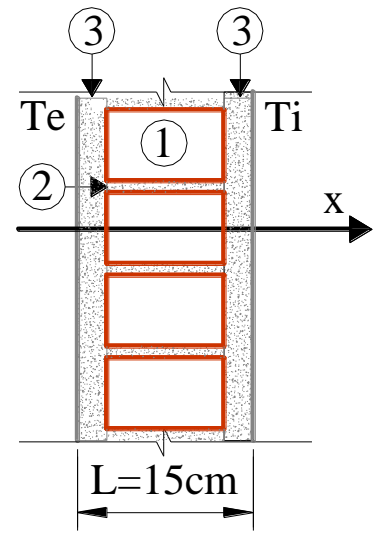

Parede 2
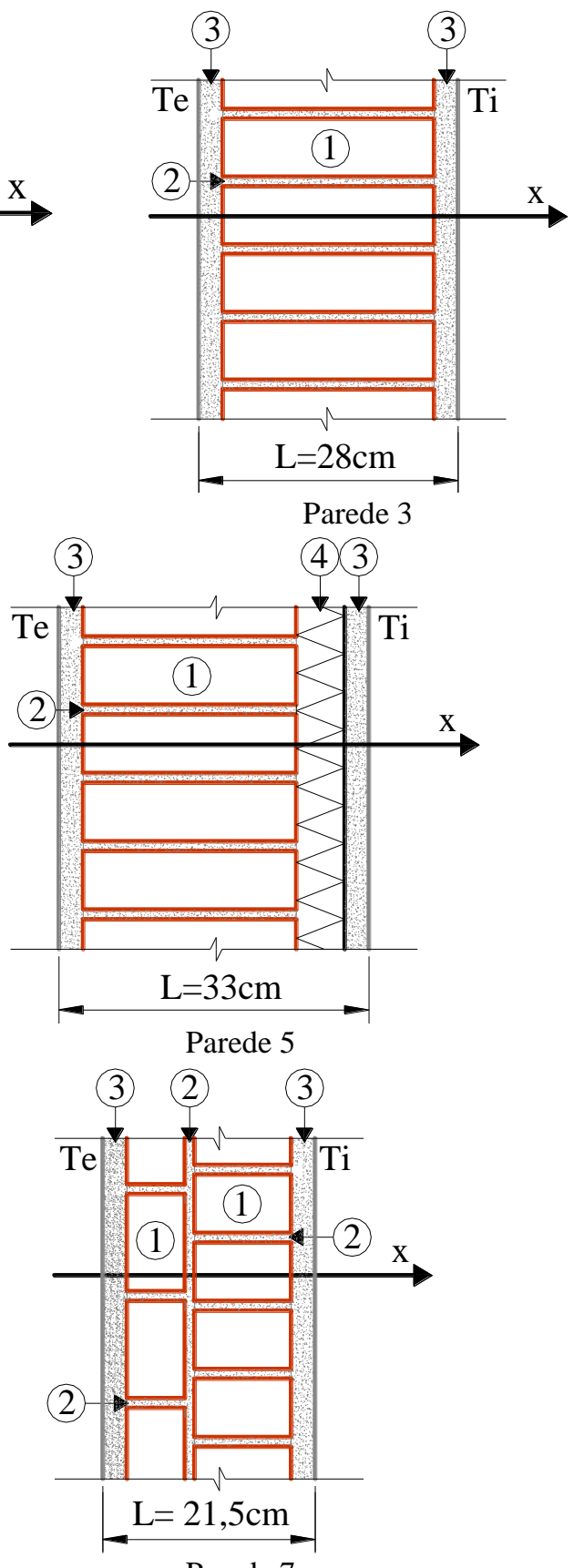

Parede 7

Legenda:

1 - Tijolo maciço cerâmico;

2 - Argamassa de assentamento;

3 - Argamassa de revestimento;

4 - Poliestireno expandido.

Figura 2 - Configurações de parede

Para efeito da modelagem, definiu-se como superfície externa da parede a superfície na qual incide o calor produzido pela lâmpada, simulando as condições ambientais da face da parede em contato com o ambiente (exterior); e como superfície interna da parede a superfície exposta às condições ambientais, simulando as condições internas do ambiente construído (interior).

\section{Transferência de calor em paredes} compostas - o Problema Direto (PD)

O problema de transferência de calor em paredes compostas por diferentes tipos de materiais foi modelado pela bem conhecida equação da condução de calor, adaptada para o caso em estudo, Equação 1, cuja demonstração pode ser 
encontrada em Incropera e Dewitt (2003) e em Özisik (1993).

$\frac{\partial T}{\partial t}=\alpha_{i} \frac{\partial^{2} T}{\partial x^{2}} \quad 0<x<L$ e $t>0$

Eq. 1

$T(0, t)=T_{l}(t)$, para $t>0$

$T(L, t)=T_{2}(t)$, para $t>0$

$T(x, 0)=T_{o}(x)$, para $0<x<L$

Onde:

$\alpha_{i}$ é a difusividade térmica $\left(\mathrm{m}^{2} / \mathrm{s}\right)$ dos materiais $i$, com $i=1,2,3,4,5$, posicionados nas partições

finitas $e_{i}$, do domínio $x \in[0, L]$;

$T$ é a temperatura $\left({ }^{\circ} \mathrm{C}\right)$;

$t$ é o tempo (s);

$x$ é a direção de transferência de calor (m);

$T_{1}(t)$ e $T_{2}(t)$ são as condições de fronteira interna e externa respectivamente; $\mathrm{e}$

$T_{o}(x)$ é a condição inicial.

A Figura 3 apresenta o detalhe de uma parede composta por diferentes materiais.

\section{Condições de contorno}

A distribuição da temperatura em um dia de sol, sem nuvens, em uma parede exposta apresenta temperaturas amenas no início e no final do dia, e um pico de temperatura máxima em torno da meia tarde. A Equação 2 foi escolhida para descrever essa evolução de temperatura em função do tempo.

As condições de fronteira foram consideradas de primeira espécie (temperatura prescrita) obtidas experimentalmente, com sensores instalados em $\mathrm{x}=0$ e $\mathrm{x}=\mathrm{L}$, para determinados valores de tempo, e expandidas para qualquer instante, por meio de um ajuste não linear. Com base nesses dados foram ajustados os parâmetros $A, a$ e $b$ da Equação 2 .

$T_{f}(t)=A e^{-a(t-b)^{2}}$

Onde:

$T_{f}$ é a temperatura nas superfícies (externa ou interna) $\left({ }^{\circ} \mathrm{C}\right)$;

$A$ é o parâmetro associado à temperatura máxima no período simulado $\left({ }^{\circ} \mathrm{C}\right)$;

$a$ é um parâmetro ajustado associado à curvatura da função (1/s);

$b$ é um parâmetro ajustado associado ao instante de máxima temperatura no período simulado (s); e

$t$ é o tempo (s).

O parâmetro $A$ está relacionado com a temperatura máxima; o parâmetro $a$ com a curvatura da função; e $b$ com o instante de tempo em que a temperatura é máxima no período. Todos os parâmetros foram ajustados pelo Método de Procura em Rede (MPR) de ajuste não linear, (SILVA NETO; MOURA NETO, 2005), através de programa próprio desenvolvido para esse fim.

A condição inicial foi obtida usando-se o valor médio de temperatura medida em cada posição da parede em $t=0 \mathrm{~s}$.

A Equação 1 foi discretizada e resolvida em Diferenças Finitas Centrais pelo Método Implícito, levando em conta as condições de contorno como funções do tempo (Equação 2) e os diferentes valores das difusividades térmicas em cada camada da parede. A solução da Equação 1, com as respectivas condições de contorno, é chamada neste trabalho de Problema Direto.

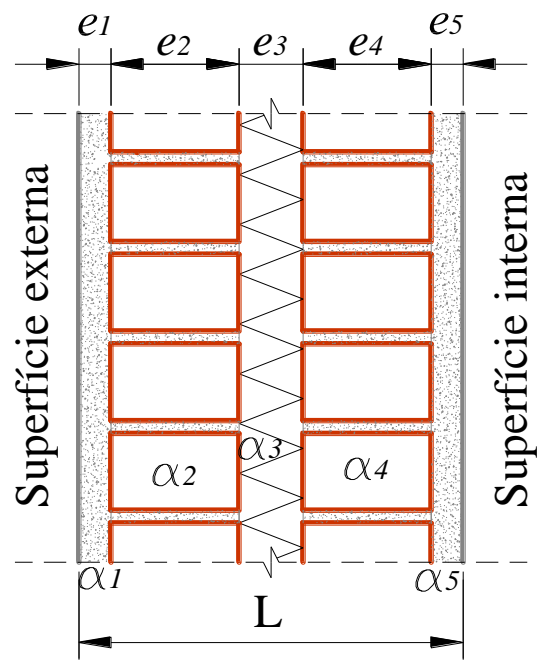

Figura 3 - Detalhe da parede composta por materiais de difusividades térmicas $a_{1}, a_{2}, \ldots, a_{5} \mathrm{e}$ espessuras $e_{1}, e_{2}, \ldots, e_{5}$ 


\section{Cálculo das difusividades térmicas - Problema Inverso (PI)}

As difusividades térmicas dos materiais foram calculadas resolvendo o problema inverso de transferência de calor, com base nos dados experimentais de temperatura em função do tempo, para cada posição dos termopares no interior da parede, utilizando-se o MPR, de acordo com os seguintes passos:

$1^{\circ}$ Passo:

Estimam-se intervalos $I_{p}=\left[\alpha_{p \min }, \alpha_{p \max }\right] \quad \mathrm{de}$ valores de difusividade onde $p=1,2,3, \ldots .5$ (número de parâmetros) que contêm, por hipótese, cada um deles, o valor ótimo da difusividade de cada material;

$2^{\circ}$ Passo:

Constrói-se uma partição de $s$ pontos $\alpha_{p k}=\alpha_{p \text { min }}+(k-1) \Delta \alpha_{p} \quad$ com $\quad k=1,2,3, \ldots, s \quad \mathrm{e}$ $\Delta \alpha_{p}=\left(\alpha_{p \max }-\alpha_{p \min }\right) /(s-1) ;$

$3^{\circ}$ Passo:

Para cada sequência de valores $\left(\alpha_{1 k}, \alpha_{2 k}, \ldots, \alpha_{n k}\right)$ para $k=1,2,3, \ldots, s$ resolve-se o Problema Direto usando a solução numérica;

$4^{\circ}$ Passo:

Calculam-se as diferenças $d_{i}$ entre as soluções calculadas $\left(T_{\text {cal }}\right)$ e os dados experimentais $\left(T_{\text {exp }}\right)$ usando a Equação 3:

$d_{i}=\sum_{j=1}^{m} \sum_{t=0}^{t f}\left(T_{c a l}(j, t)-T_{\exp }(j, t)\right)^{2}$

Onde:

$i=1,2,3, \ldots, s^{n}$;

$T_{\exp }(j, t)$ são os dados experimentais para cada posição $j$ e tempo $t$; e

$5^{\circ}$ Passo:

Identifica-se o menor valor de $d_{i}\left(d_{\min }\right)$. Essa diferença corresponde ao conjunto de parâmetros $\alpha_{o t}$ para o intervalo $I_{p}$.

O MPR é um método que não tem problemas de convergência, pois busca por inspeção direta a solução que apresenta a menor diferença $d_{i}$ (Equação 3) entre um conjunto de soluções finito. Por esse motivo pode ser considerado um método de soluções subótimas, porém, para um número suficientemente grande de divisões, em que o coeficiente de correlação não apresenta variações significativas, os resultados tendem a soluções ótimas. O método tem a vantagem de não necessitar de cálculo de derivadas, porém sua execução computacional tende a ser mais demorada que outros métodos, como LevenbergMarquardt e Gradientes Conjugados, apresentados em Özisik e Orlande (2000) e em Silva Neto e Moura Neto (2005), por exemplo. Mesmo assim, mostrou-se um método eficiente para o presente problema.

O cálculo da difusividade, em que são utilizados os problemas direto e inverso, foi realizado numericamente com programa desenvolvido e executado no software MATLAB.

\section{Cálculo dos fluxos de calor nas superfícies das paredes}

O fluxo de calor em uma superfície $S$, em dado instante de tempo, é definido pela Lei de Fourier, que discretizada, pode ser escrita com a Equação 4:

$q=k A \frac{T_{2}-T_{1}}{x_{2}-x_{1}}$

Eq. 4

Onde:

$q$ é o fluxo de calor $\left(\mathrm{Wm}^{-2}\right)$;

$A$ é a área, nesse caso considerada unitária $\left(\mathrm{m}^{2}\right)$

$k$ é a condutividade térmica $\left(\mathrm{Wm}^{-10} \mathrm{C}^{-1}\right)$;

$x_{1}$ e $x_{2}$ são posições nas superfícies à esquerda e à direta da superfície respectivamente; e

$T_{1}$ e $T_{2}$ são as temperaturas nas posições $x_{1}$ e $x_{2}$.

Neste trabalho foram utilizados os valores de condutividade térmica da literatura (INCROPERA; DEWITT, 2003).

A quantidade total de calor que passa, por metro quadrado, pela superfície $S$ durante o tempo de experimento foi obtida somando-se o produto do fluxo de calor pelo intervalo de tempo (Equação 5):

$Q_{T_{j}}=\sum_{i=1}^{n} k_{j} \frac{T_{2 i}-T_{1 i}}{x_{2 i}-x_{1 i}} d t$

Eq. 5

Onde:

$Q_{T}$ é a quantidade total de calor $\left(\mathrm{Jm}^{-2}\right)$ no período de experimento;

$d t$ é o intervalo de tempo (s);

$n$ é o número de intervalos de tempo;

os subíndices 1 e 2 referem-se às posições interna ou externa à superfície considerada; e

j é a superfície cujo fluxo está sendo calculado. 
É importante observar que a variável $Q_{T}$ pode assumir valores positivos ou negativos. $\mathrm{Na}$ superfície $x=0$ (externa), se $Q_{T}<0$, significa que a parede recebeu calor do ambiente no período de experimento. Na superfície $x=L$ (interna), se $Q_{T}<$ 0 , significa que a parede transferiu calor para o ambiente no período de experimento. Portanto, quanto menor o valor de $Q_{T}$, pior será seu desempenho como parede isolante.

\section{Relação termoeconômica}

Com o intuito de avaliar o desempenho térmico e econômico das estruturas estudadas, criou-se o índice $E$ (Equação 6), que é a relação termoeconômica por unidade de área construída de parede.

$E=\left(\frac{Q_{T}}{N C}\right)$

Eq.6

Onde:

$E$ é a relação termoeconômica por metro quadrado de parede (adimensional);

$C$ é o custo total de cada parede por metro quadrado $\left(R \$ / m^{2}\right)$; e

$N$ é um valor de $Q_{T} / C$ arbitrário, relativo a uma parede com péssima relação termoeconômica.

Neste trabalho usou-se o valor de $N=-1,2 \times 10^{5}$ $J R \$^{-2}$. O valor de $N$ foi definido com base no menor valor observado da razão $Q_{T} / C$ para os casos estudados, acrescido de uma margem extra, pois podem existir paredes com essa razão inferior aos casos estudados. Dessa forma, a relação termoeconômica fica definida no intervalo $-\infty<E$ $<1$, sendo que o valor 1 refere-se a uma parede de péssimo desempenho termoeconômico; se $0<E<$ 1 , a parede cede calor ao ambiente interno, e se $-\infty$ $<E<0$, a parede retira calor do ambiente interno.

\section{Análise dos resultados}

Os resultados do problema inverso sobre a estimação das difusividades térmicas dos materiais das paredes foram utilizados para determinar a distribuição de temperatura em função do tempo e do espaço em cada parede. Essa distribuição foi comparada com os dados experimentais, e com ela foi possível calcular os fluxos de calor nas superfícies e determinar a relação termoeconômica de cada parede.

\section{Estimação das difusividades térmicas dos materiais}

Os resultados do problema inverso para o cálculo das difusividades térmicas dos materiais constituintes de cada parede são apresentados na Tabela 1, com os respectivos coeficientes de correlação. Observa-se uma pequena variação entre as difusividades do mesmo material, porém todas estão na mesma ordem de grandeza, inclusive de acordo com dados da literatura (INCROPERA; DEWITT, 2003). Essas variações, apesar de existentes, não são significativas para o cálculo do fluxo de calor e análise do desempenho termoeconômico das paredes, objetivo deste trabalho. Por isso, são adotadas as difusividades apresentadas na Tabela 1 .

Os resultados do Problema Direto com os dados da Tabela 1, juntamente com os dados experimentais, são apresentados nas Figuras 4 e 5 para as Paredes 4 e 6 , onde se pode observar a significativa correspondência entre os resultados calculados e experimentais, reforçada pelos valores próximos a 1 do coeficiente de correlação (Tabela 1). As demais paredes apresentaram resultados semelhantes.

\begin{tabular}{c|c|c|c|c}
\hline Paredes & $\begin{array}{c}\text { Reboco }\left(\mathbf{m}^{2} / \mathbf{s}\right) \\
\left(\mathbf{x ~ 1 0} \mathbf{1 0}^{-7}\right)\end{array}$ & $\begin{array}{c}\text { Tijolo }\left(\mathbf{m}^{\mathbf{2}} / \mathbf{s}\right) \\
\left(\mathbf{x ~ \mathbf { 1 0 } ^ { - 7 } )}\right.\end{array}$ & $\begin{array}{c}\text { Poliestireno }\left(\mathbf{m}^{2} / \mathbf{s}\right) \\
(\mathbf{x ~ 1 0})\end{array}$ & $\mathbf{R}^{\mathbf{2}}$ \\
\hline $\mathbf{1}$ & 3,50 & 4,00 & - & 0,67 \\
\hline $\mathbf{2}$ & 3,47 & 3,40 & - & 0,98 \\
\hline $\mathbf{3}$ & 5,00 & 6,87 & - & 0,97 \\
\hline $\mathbf{4}$ & 4,36 & 6,33 & 4,76 & 0,91 \\
\hline $\mathbf{5}$ & 4,33 & 5,53 & 5,00 & 0,97 \\
\hline $\mathbf{6}$ & 4,33 & 5,53 & 5,00 & 0,94 \\
\hline $\mathbf{7}$ & 5,53 & 3,67 & - & 0,97 \\
\hline
\end{tabular}

Tabela 1 - Difusividades térmicas dos materiais 


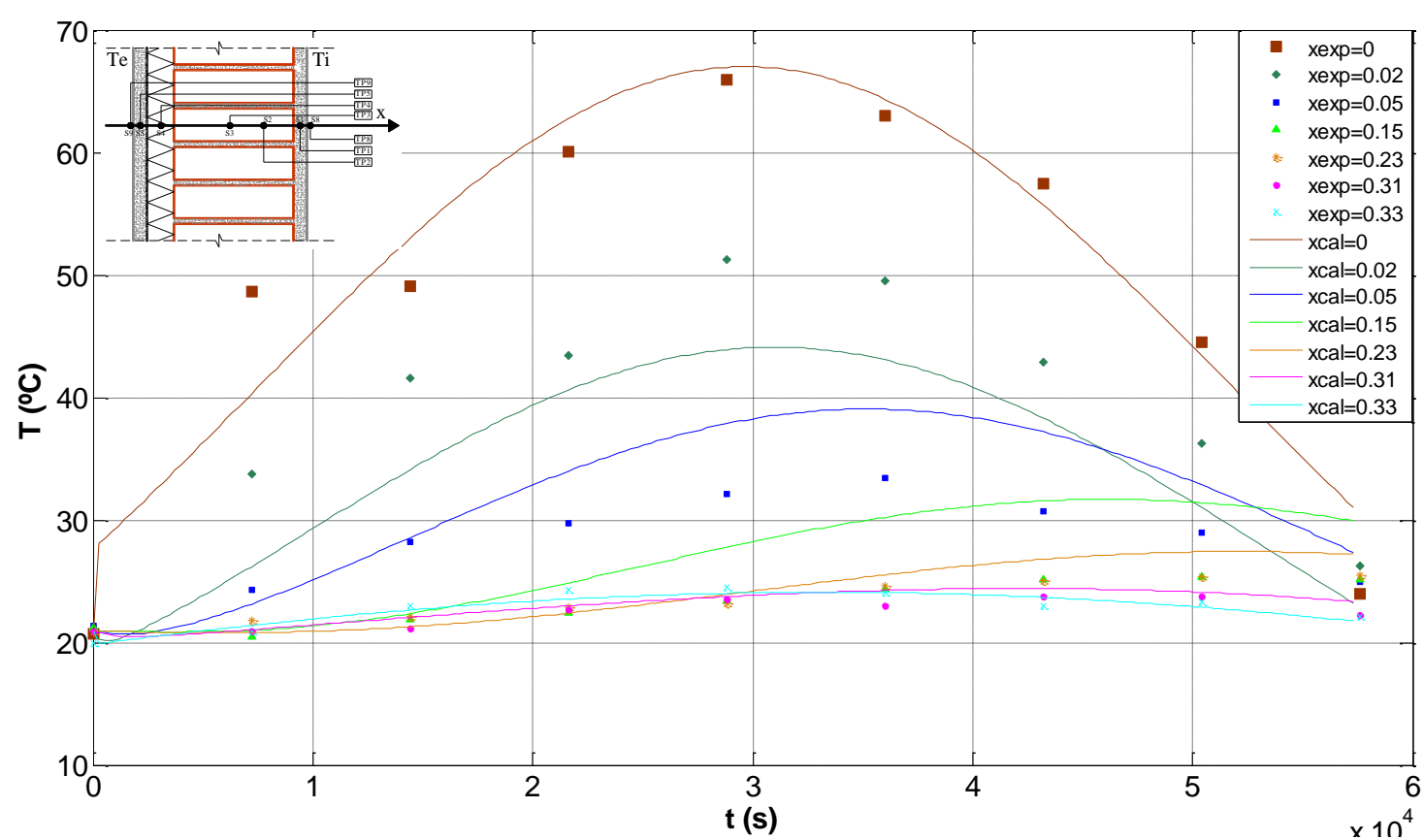

Figura 4 - Comparação dos dados experimentais com os resultados calculados com as difusividades da Tabela 1 para a Parede 4

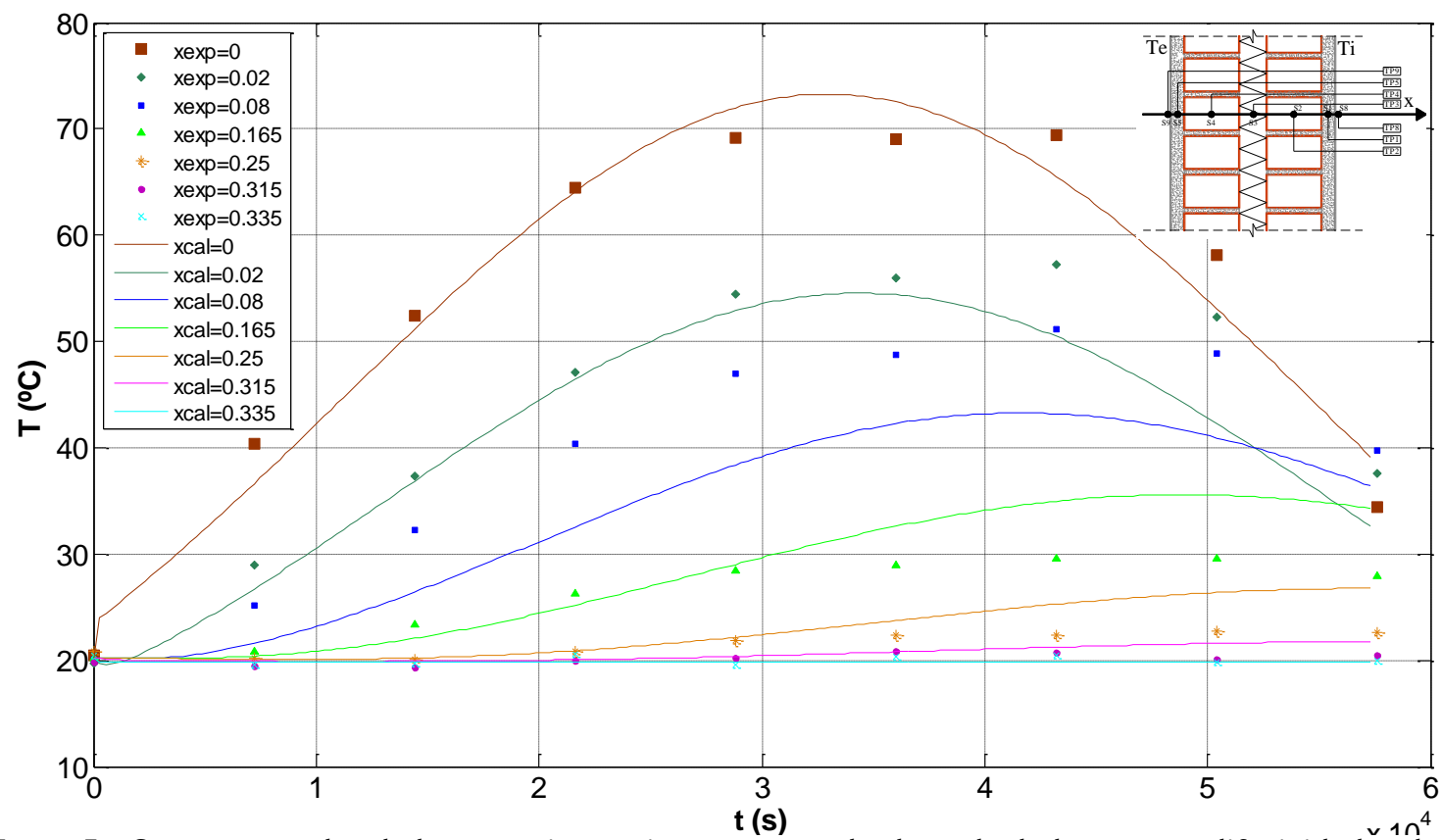

Figura 5 - Comparação dos dados experimentais com os resultados calculados com as difusividades da Tabela 1 para a Parede 6

\section{Fluxo de calor}

O cálculo do fluxo de calor na superfície externa $(\mathrm{x}=0)$ e na superfície interna $(\mathrm{x}=\mathrm{L})$ da parede em função do tempo permite analisar as trocas de calor da parede com o meio. A Figura 6 apresenta esses resultados para a Parede 4 , onde se observa que as trocas com o ambiente interno são mínimas e que nas 8 primeiras horas há uma tendência da parede de retirar calor do ambiente, enquanto nas 8 últimas horas a parede cede calor. Essa análise é complementar a análise da quantidade de calor total trocada através da superfície, pois especifica os períodos em que há aquecimento/resfriamento do ambiente interno. 


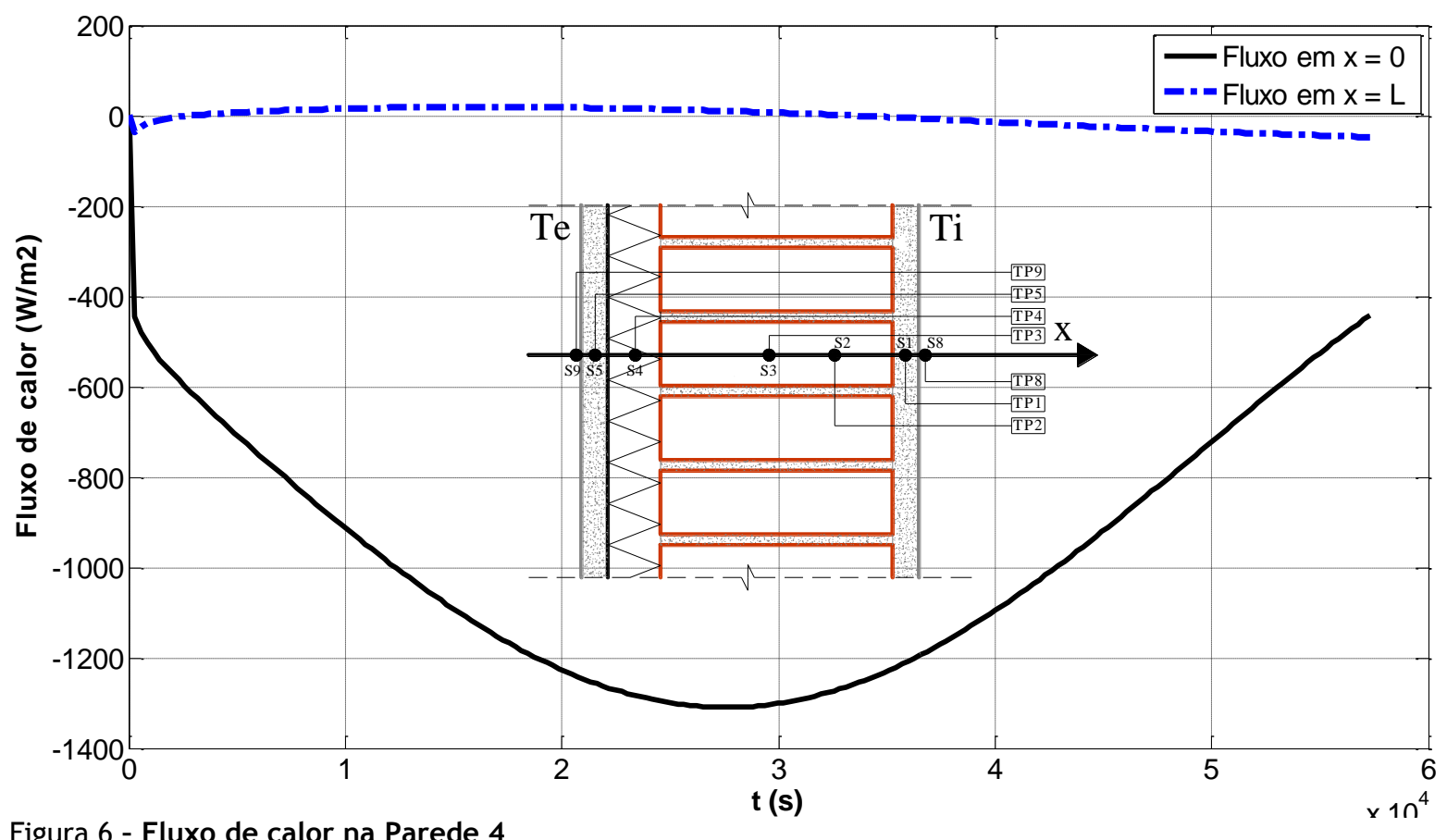

Figura 6 - Fluxo de calor na Parede 4

\begin{tabular}{|c|c|c|c|c|c|c|}
\hline \multirow[b]{2}{*}{ Parede } & \multicolumn{3}{|c|}{$x=0$} & \multicolumn{3}{|c|}{$x=L$} \\
\hline & $\begin{array}{c}\underset{\text { Fmax }}{\left(\mathbf{W} / \mathbf{m}^{2}\right)} \\
\end{array}$ & $\begin{array}{c}\text { Tempo } \\
\text { Fmax (h) }\end{array}$ & $\begin{array}{c}\text { QT x 10 }{ }^{7} \\
\left(\mathrm{~J} / \mathbf{m}^{2}\right)\end{array}$ & $\begin{array}{c}\underset{\text { Fmax }}{\left(\mathbf{W} / \mathbf{m}^{2}\right)} \\
\end{array}$ & $\begin{array}{c}\text { Tempo } \\
\text { Fmax (h) }\end{array}$ & $\begin{array}{c}\text { QT x 10 }{ }^{7} \\
\left(\mathrm{~J} / \mathbf{m}^{2}\right)\end{array}$ \\
\hline 1 & $-354,38$ & 6,00 & $-0,93$ & $-202,61$ & 11,60 & $-61,73$ \\
\hline 2 & $-908,93$ & 8,00 & $-3,73$ & $-117,43$ & 12,72 & $-27,90$ \\
\hline 3 & $-1160,77$ & 7,60 & $-4,78$ & $-63,36$ & 0,08 & $-10,04$ \\
\hline 4 & $-1309,66$ & 7,68 & $-5,63$ & $-47,19$ & 15,92 & $-2,38$ \\
\hline 5 & $-924,66$ & 8,16 & $-3,88$ & $-68,13$ & 0,08 & $-11,83$ \\
\hline 6 & $-1067,25$ & 8,24 & $-4,47$ & $-55,05$ & 15,92 & $-12,46$ \\
\hline 7 & $-1107,71$ & 8,08 & $-4,63$ & $-76,04$ & 0,08 & $-15,11$ \\
\hline
\end{tabular}

Tabela 2 - Fluxo e quantidade de calor para $16 \mathrm{~h}$

Conhecidos os fluxos de calor em $\mathrm{x}=0$ e em $\mathrm{x}=\mathrm{L}$ para todas as configurações de parede, foi possível obter os fluxos máximos (Fmax), bem como a quantidade de calor (QT) de cada parede nas 16 horas de duração do ensaio (Tabela 2).

O fluxo máximo é um dado importante para estudar o conforto térmico e a capacidade de isolamento das paredes. Paredes que evitam um pico alto de fluxo tendem a manter as condições do ambiente menos variáveis termicamente.

A quantidade total de calor $Q_{T}$ (Equação 5) dá o balanço geral de calor na superfície considerada, portanto, se $Q_{T}$ é nula, não significa que não houve troca de calor, mas que as quantidades de calor cedida e retirada do ambiente são idênticas. Para os casos estudados, todas as paredes apresentaram $Q_{T}$ negativo na superfície interna, portanto todas cedem calor para o ambiente interno no período considerado, porém com sensíveis diferenças entre uma e outra parede.

A análise do valor de $Q_{T}$ na superfície interna ( $x=$ $L)$ indica a eficiência da parede como isolante térmico. Paredes com valores absolutos de $Q_{T}$ próximos a zero são eficientes como isolantes, pois minimizam a carga térmica dos ambientes internos. Com esse critério, a parede que deixa passar menor quantidade de calor, isto é, a que apresenta melhor desempenho térmico, é a Parede 4, e a que possui o pior desempenho térmico é a Parede 1, como mostra a Tabela 2.

\section{Análise termoeconômica}

A composição do custo total por metro quadrado de cada parede foi realizada com base nos preços de insumos e de serviços do SINAPI (SISTEMA..., 2009), e os resultados são apresentados na Figura 7. 


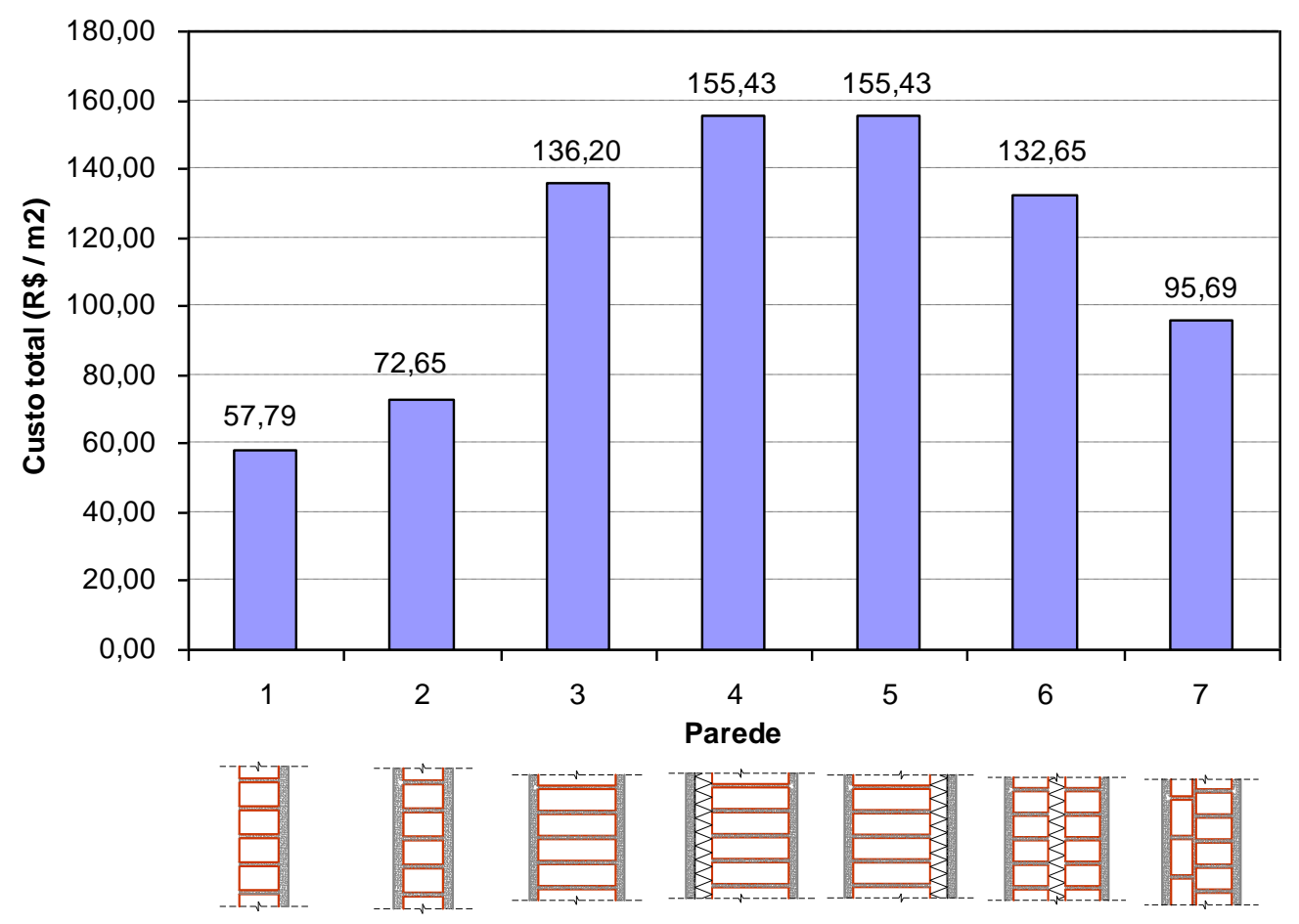

Figura 7 - Custo total por metro quadrado das paredes estudadas

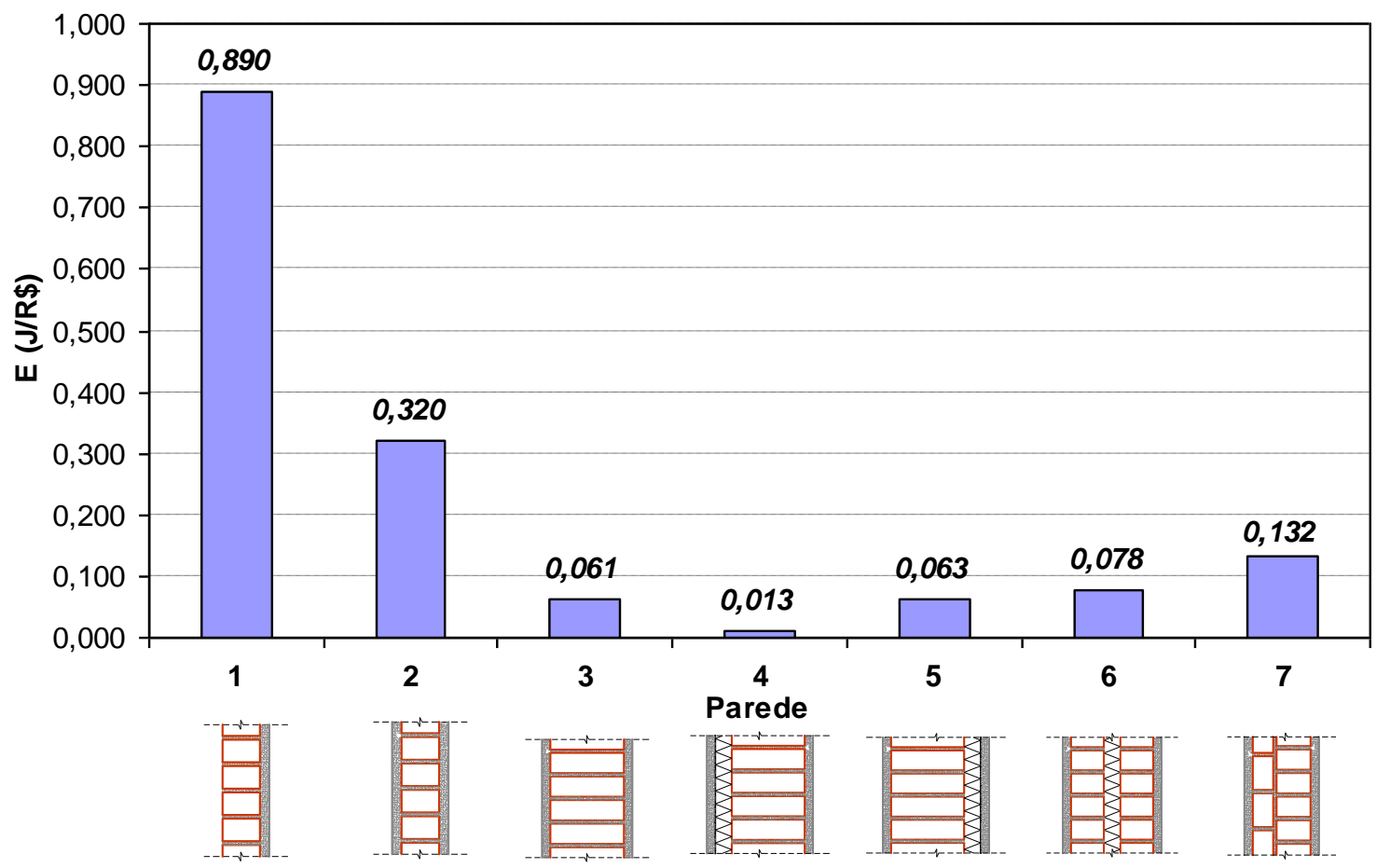

Figura 8 - Relação termoeconômica das paredes

Aplicando-se a Equação 5 com os valores correspondentes de quantidade de calor $Q_{T}$ em $x=$ $L$, da Tabela 2, e o custo total $C$, dado pela Figura 7, obtêm-se a relação termoeconômica de cada parede (Equação 6), apresentada na Figura 8.
Pela análise do gráfico, fica evidente quais paredes tiveram a melhor relação termoeconômica. A Parede 4 é a parede com melhor desempenho, apesar de apresentar o maior custo total. $\mathrm{O}$ baixo $\mathrm{E}$ é justificado por esta apresentar o isolamento térmico pelo exterior, impedindo a entrada de calor 
já nas primeiras camadas e minimizando a condução do calor até a superfície interna.

Nas condições dos experimentos, o segundo melhor desempenho termoeconômico é o da Parede 3. Na sequência, as Paredes 5 e 6 possuem índice $E$ semelhante (neste caso, se tivesse que se escolher entre ambas as paredes, indicar-se-ia a Parede 3 para lugares onde é necessária maior inércia térmica, e a Parede 5 para determinados casos onde o que importa é manter a temperatura interior constante - câmara fria, silos). Dessa forma, recomenda-se utilizar as configurações das Paredes 3 a 6 (alto desempenho térmico e econômico) nas edificações, para reduzir gastos com sistemas de calefação/refrigeração, melhorando o conforto térmico e a eficiência energética.

Pode-se classificar a Parede 7 como sendo uma parede com um médio desempenho termoeconômico, ficando entre as paredes com alto desempenho termoeconômico e as paredes com baixo índice E. Esta parede é uma alternativa para situações em que a questão térmica não é tão relevante (depósitos, garagens, banheiros, etc.).

Já entre as paredes de baixo desempenho está a Parede 2, que é a configuração de parede mais utilizada em edificações comuns. Por fim, a Parede 1 possui o pior desempenho termoeconômico, mesmo sendo a parede com menor custo total. Não se recomenda utilizar essas paredes para o clima brasileiro, pois causariam desconforto nos ocupantes das edificações.

\section{Conclusões}

Com base nos resultados obtidos nesta pesquisa algumas conclusões podem ser feitas.

Através dos resultados dos ensaios experimentais, realizados na câmara térmica, foi possível medir a transferência de calor de diferentes configurações de paredes ao longo do tempo.

O método de determinação das difusividades térmicas dos materiais constituintes de cada parede mostrou-se eficiente. Os valores das difusividades térmicas dos materiais estudados encontram-se dentro da seguinte faixa de valores:

Reboco: 3,50 a $5,53 \times 10^{-7}\left(\mathrm{~m} / \mathrm{s}^{2}\right)$;

Tijolo: 3,40 a $6,86 \times 10^{-7}\left(\mathrm{~m} / \mathrm{s}^{2}\right)$; e

Poliestireno: 4,75 a 5,00 ×10 $10^{-7}\left(\mathrm{~m} / \mathrm{s}^{2}\right)$.

A comparação dos fluxos e das quantidades de calor para cada composição é uma informação importante para a análise do desempenho térmico, visando a uma melhor utilização dos materiais e à minimização do gasto de energia com refrigeração e aquecimento de ambientes.

A avaliação das diferentes configurações de estruturas foi realizada através da relação entre o desempenho térmico e econômico de cada parede, conforme sumarizado na Figura 9.

Essa avaliação não dispensa a análise caso a caso de cada edificação - continua sendo necessário estudar as demais variáveis intervenientes no ambiente construído (variáveis climáticas, humanas e arquitetônicas) -, mas através dela se destaca a importância que uma análise termoeconômica (e não só econômica) tem para apontar possíveis soluções construtivas que podem ser utilizadas, com o intuito de melhorar o desempenho energético das edificações. Também é importante frisar que, muitas vezes, edificações que adotam soluções construtivas que requerem baixo investimento inicial, no longo prazo, consomem muito mais energia, consequentemente com custos financeiros muito maiores do que edificações mais eficientes energeticamente, que requerem um investimento inicial maior.

O programa desenvolvido é um instrumento útil para a escolha de soluções construtivas, visando melhorar a eficiência energética das edificações; permite a simulação do desempenho térmico e econômico de outras configurações de paredes (além das estudadas neste trabalho), sem a necessidade de construí-las, desde que se conheçam os valores das difusividades térmicas e os custos dos materiais utilizados.

\begin{tabular}{|c|c|c|c|c|}
\hline $\begin{array}{c}\text { Desempenho } \\
\text { termo-econômico }\end{array}$ & $\begin{array}{c}\text { Classificação } \\
\text { geral }\end{array}$ & Parede & Configuração (cm) & $\begin{array}{l}\text { Espessura } \\
\text { Total }(\mathbf{c m})\end{array}$ \\
\hline \multirow{4}{*}{ Alto } & $1^{\circ}$ & $\mathrm{P} 4$ & reboco $(2,5)+$ EPS $(5)+$ tijolo $(23)+$ reboco $(2,5)$ & 33,0 \\
\hline & $2^{\circ}$ & $\mathrm{P} 3$ & reboco $(2,5)+$ tijolo $(23)+$ reboco $(2,5)$ & 28,0 \\
\hline & $3^{\circ}$ & P5 & reboco $(2,5)+$ tijolo $(23)+\operatorname{EPS}(5)+$ reboco $(2,5)$ & 33,0 \\
\hline & $4^{\circ}$ & P6 & $\begin{array}{l}\text { reboco }(2,5)+\text { tijolo }(11,5)+\text { EPS }(5,5)+\text { tijolo } \\
(11,5)+\text { reboco }(2,5)\end{array}$ & 33,5 \\
\hline Médio & $5^{\circ}$ & P7 & reboco $(2,5)+$ tijolo $(16,5)+$ reboco $(2,5)$ & 21,5 \\
\hline \multirow[t]{2}{*}{ Baixo } & $6^{\circ}$ & $\mathrm{P} 2$ & reboco $(2,5)+$ tijolo $(10)+$ reboco $(2,5)$ & 15,0 \\
\hline & $7^{\circ}$ & P1 & tijolo $(10,5)+$ reboco $(2,5)$ & 13,0 \\
\hline
\end{tabular}

Figura 9 - Desempenho termoeconômico 


\section{Referências}

BECKETT, R. E.; CHU, S. C. Finite Element Method Applied to Heat Conduction in Solids with Monlinear Boundary Conditions. Journal of Heat Transfer, v. 95, p. 126-129, 1973.

CARSLAW, H. S.; JAEGER, J. C. Conduction of Heat in Solids. 2. ed. London: Oxford University Press, 1959.

CHEN, H. T.; LIN, J. Y. Application of the Hybrid Method to Transient Heat Conduction in OneDimensional Composite Layers. Computer \& Structures, v. 39, n 5, p. 451-458, 1991.

CHLELA, F. et al. A Statistical Method to Improve the Energy Efficiency of an Office Building. In: BUILDING SIMULATION, Beijing, 2007. Proceedings... Beijing, 2007. p. 1756-1764.

CHONG, W.; TRAMONTINI, R.; SPECHT, L. P. Application of the Laplace Transform and Its Numerical Inversion to Temperature Profile of a Two-Layer Pavement Under Site Conditions. Numerical Heat Transfer, Part A, Applications, v. 55, n. 11, p. 1004-1018, 2009.

\section{EUROACE. Towards Energy Efficient}

Buildings in Europe. Final report June, 2004.

HUANG, S. C.; CHANG, Y. P. Heat Conduction in Unsteady, Periodic and Steady States in Laminated Composites. Journal of Heat Transfer, v. 102, p. 742-748, 1980.

INCROPERA, F. P.; DE WITT, D. P. Fundamentos de Transferência de Calor e de Massa. 5. ed. Rio de Janeiro: LTC, 2003. 698 p.

ITARD, L.; MEIJER, F. Towards a Sustainable Northern European Housing Stock: figures, facts and future. [s.1.]: IOS Press, 2008.

KWOK, A. G.; RAJKOVICH, N. B. Addressing Climate Change in Comfort Standards. Building and Environment, v. 45, n. 1, p. 18-22, 2009.

LAMBERTS, R.; DUTRA, L.; PEREIRA, F. O. R. Eficiência Energética na Arquitetura. São Paulo: PW, 1997. 188 p.

ÖZIŞIK, M. N. Heat Conduction. New York: John Wiley \& Sons, 1993. 273 p.

ÖZIŞIK, M. N.; ORLANDE, H. R. B. Inverse Heat Transfer: fundamentals and applications. New York: Taylor Francis, 2000. 330 p.
PEÑA, C. C.; GHISI, E.; PEREIRA, C. D. Comparação entre Necessidade e Disponibilidade de Vento e Radiação Solar para Fins de Análise Bioclimática de Edificações em Florianópolis. Ambiente Construído, Porto Alegre, v. 8, n. 4, p. 87-101, out./dez. 2008.

PROGRAMA NACIONAL DE CONSERVAÇÃO DE ENERGIA ELÉTRICA. Disponível em: <http://www.procel.gov.br/>. Acesso em: 19 abr. 2009.

RUPP, R. F. Análise da Transferência de Calor em Paredes Compostas por Tijolos Maciços. 2009. 119 f. Trabalho de Conclusão de Curso (Curso de Engenharia Civil) - Universidade Regional do Noroeste do Estado do Rio Grande do Sul, 2009.

SANTIN, O. G.; ITARD, L.; VISSCHER, H. The Effect of Occupancy and Building Characteristics on Energy Use for Space and Water Heating in Dutch Residential Stock. Energy and Buildings, v. 41, n. 11, p. 1223-1232, 2009.

SILVA NETO, A. J.; MOURA NETO, F. D. Problemas Inversos: conceitos fundamentais e aplicações. Rio de Janeiro: UERJ, 2005. 172 p.

SISTEMA NACIONAL DE PREÇOS E ÍNDICES PARA A CONSTRUÇÃO CIVIL. Relatórios de Insumos e de Serviços por Estado. Disponível em:

<http://www1.caixa.gov.br/download/index.asp>. Acesso em: 09 out. 2009.

SUGIYAMA, S.; NISHIMURA, M.; WATANABE, H. Transient Temperature Response of Composite Solids. International Journal of Heat and Mass Transfer, v. 17, p. $875-883,1974$

SUN, L.; JIA, L.; HUANG, L. Numerical Temperature Prediction Model and Thermal Properties for Asphalt Pavement. In: LOIZOS; SCARPAS; AL-QADI (Ed.). Advanced Characterisation of Pavement and Soil Engineering Materials. London: Taylor \& Francis Group, 2007.

\section{Agradecimento}

Ao MEC, pela bolsa PET concedida ao primeiro e terceiro autores. 